\section{Efecto de los antiinflamatorios no esteroideos AINEs, analgésicos y coxibs sobre la magnitud del movimiento dentario ortodóntico}

\author{
Effect of nonsteroidal antiinflammatory drugs NSAIDs, analgesics \\ and coxibs on the magnitude and rate of orthodontic tooth \\ movement
}

\begin{abstract}
Resumen
El objetivo del presente estudio fue determinar los efectos de diclofenaco, etoricoxib, ibuprofeno y paracetamol sobre la magnitud del movimiento dentario ortodóntico. Participaron 50 ratas Holtzman distribuidas en 5 grupos de 10 ratas cada uno. Al inicio del trabajo, en cada uno de los animales, se midió la distancia entre los ángulos incisodistales de los incisivos maxilares empleando un calibrador, e inmediatamente se adhirió un dispositivo ortodóntico con una fuerza de acción recíproca de $35 \mathrm{~g}$ en dichos incisivos. Cada grupo recibió tratamientos diferentes por vía intraperitoneal $(\mathrm{A}: \mathrm{NaCl}$, grupo control, B: diclofenaco, C: etoricoxib, D: ibuprofeno, E: paracetamol) durante 5 días. En todos los controles hechos al 1er, 3er, 5 to y $7 \mathrm{mo} \mathrm{día,} \mathrm{la} \mathrm{distancia} \mathrm{interincisiva} \mathrm{fue} \mathrm{significativamente} \mathrm{menor} \mathrm{en} \mathrm{los}$ grupos ibuprofeno y diclofenaco, y sin diferencia significativa en los grupos etoricoxib y paracetamol; comparados todos con el grupo control. Se demostró que la administración de ibuprofeno y diclofenaco disminuyen significativamente la magnitud del movimiento dentario ortodóntico en ratas. Se demostró que la administración de paracetamol y etoricoxib no disminuyen significativamente la magnitud del movimiento dentario ortodóntico en ratas.
\end{abstract}

Palabras claves: Movimiento dentario ortodóntico, Anti-inflamatorios no esteroideos, AINEs, analgésicos, coxibs

\begin{abstract}
The objective of this study was to determine the effects of diclofenac, etoricoxib, ibuprofen and paracetamol on the magnitude of orthodontic tooth movement. 50 Holtzman rats participated in 5 groups of 10 rats each. At the beginning of study the distance between the angles inciso-distals of both maxillary incisive was measured in each animal, using a caliper, and immediately an orthodontic device with a reciprocal action force of $35 \mathrm{~g}$. was adhered to them. Each group received different treatments intraperitoneally (A: $\mathrm{NaCl}$, control group, B: diclofenac, C: etoricoxib, D: ibuprofen, E: paracetamol) for 5 days. In all controls made the $1 \mathrm{st}, 3 \mathrm{rd}$, 5 th and 7 th days, interincisive distance was significantly lower in the ibuprofen and diclofenac groups, without significant difference in the etoricoxib and paracetamol groups; all compared with control group. It was shown that administration of ibuprofen and diclofenac significantly diminish the magnitude of orthodontic tooth movement in rats. It was demonstrated that administration of paracetamol and etoricoxib not significantly decrease the magnitude of orthodontic tooth movement in rats.
\end{abstract}

Keywords: Orthodontic tooth movement, Non-steroidal anti-inflammatory drugs, NSAIDs, analgesics, coxibs

\section{Artículo Original}

\begin{abstract}
Víctor Manuel Chumpitaz Cerrate ${ }^{1,2}$, Luciano Soldevilla Galarza ${ }^{3}$, Vilma Chuquihuaccha Granda', Adrián Mallma Medina ${ }^{2}$, Martha Rodríguez Vargas ${ }^{4}$, Franco Tauquino Alvarez ${ }^{5}$, Eliberto Ruiz Ramírez6, César Franco Quino ${ }^{6}$, Elías Aguirre Siancas $^{6}$, Juan Eche Herrera ${ }^{6}$, Yuri Castro Rodríguez,
\end{abstract}

1. Laboratorio de Fisiología y Farmacología, Facultad de Odontología, Universidad Nacional Mayor de San Marcos, Lima, Perú.

2. Departamento Académico de Ciencias Básicas.

3. Departamento Académico de Estomatología Pediátrica.

4. Departamento Académico de Estomatología Preventiva y social.

5. Departamento Académico de Estomatología Rehabilitadora.

6. Estudiante de pregrado Odontologia.

1-6. Facultad de Odontología, Universidad Nacional Mayor de San Marcos, Lima, Perú.

\section{Correspondencia:}

Dr. Víctor Manuel Chumpitaz Cerrate

Laboratorio de Fisiología y Farmacología, Facultad de Odontología, Universidad Nacional Mayor de San Marcos. Av. Germán Amézaga s/n, Lima 1. Perú.

Correo electrónico: vchumpitazc@unmsm.edu.pe

Fecha recepción: 25-02-14

Fecha aprobación: 19-06-14

\section{Introducción}

El movimiento dentario ortodóntico MDO se caracteriza por cambios en la remodelación de los tejidos dentales y paradentales, que se originan por la creación de áreas de compresión y tensión en el ligamento periodontal. El MDO puede ocurrir rápida o lentamente, dependiendo de las características físicas de las fuerzas aplicadas, del tamaño y respuesta del ligamento periodontal. Las modificaciones de las fuerzas aplicadas alteran la vascularidad y el flujo sanguíneo periodontal, determinando la síntesis y liberación local de neurotransmisores, citoquinas, factores de crecimiento, factores estimulantes de colonias y metabolitos del ácido araquidónico. Estas moléculas provocan numerosas respuestas de diversos tipos celulares en y alrededor de las piezas dentarias, produciendo un microambiente favorable para la reabsorción o aposición tisular. ${ }^{1,2}$

El daño celular o la alteración no destructiva de la membrana producido por la aplicación de fuerzas ortodónticas activan la fosfolipasa, la cual induce la liberación de prostaglandinas (PGs). Las PGs cumplen un rol importante como agentes reguladores de la remodelación 
ósea inducida por el stress mecánico. Las PGs de la serie E y $F$ han sido implicadas en acciones de remodelación ósea, particularmente reabsorción; disminuyendo la síntesis de colágeno e incrementando el AMPc, favoreciendo el MDO. Se ha demostrado un incremento en el número de osteoclastos luego de la inyección local de PGs en los tejidos paradentales de ratas. ${ }^{2,3,4,5}$

El tratamiento de ortodoncia puede provocar sensaciones desagradables e incluso dolorosas. Los ortodoncistas, frecuentemente, prescriben fármacos analgésicos a sus pacientes para aliviar el dolor, el cual disminuye usualmente antes de 7 días luego de la activación. Muchos de estos fármacos son antiinflamatorios no esteroideos AINEs. Hay evidencia en estudios en animales que el MDO aumenta significativamente ante la aplicación de PGs. Por otra parte se ha reportado que la indometacina y el flurbiprofeno - inhibidores específicos de la síntesis de PGs - redujeron el número de osteoclastos en el hueso alveolar de gatos y conejos que recibieron fuerzas ortodónticas y esto redujo el MDO hasta un 50\%. .,7 $^{6}$

Diversos estudios que evalúan el efecto de los analgésicos sobre el MDO muestran resultados similares en algunos casos y distintos en otros. ${ }^{8}$ En un estudio en ratas donde compararon diclofenaco $(10 \mathrm{mg} / \mathrm{kg})$ y rofecoxib $(1 \mathrm{mg} /$ $\mathrm{kg}$ ) observaron que ambos fármacos inhibieron significativamente el MDO, parcialmente rofecoxib y totalmente diclofenaco. ${ }^{9}$ En un estudio similar realizado en ratas donde compararon el efecto de los coxibs, se encontró que el rofecoxib interfiere con el MDO, mientras no se observó interferencia con parecoxib y celecoxib. ${ }^{10}$ Otra investigación en animales encontró que la terapia con celecoxib inhibió el MDO. ${ }^{11}$

El modelo murino de MDO es el más utilizado, universalmente aceptado, ético y posible de extrapolación para el hombre. ${ }^{12}$ Un trabajo realizado en ratas para determinar los efectos del ácido acetilsalicílico $(100 \mathrm{mg} / \mathrm{kg})$, ibuprofeno $(30 \mathrm{mg} / \mathrm{kg}$ ) y acetaminofén $(200 \mathrm{mg} /$ $\mathrm{kg}$ ) sobre el MDO inducido por un resorte colocado en los incisivos maxilares, evidenció que el ácido acetil salicílico y el ibuprofeno disminuyeron el número de osteoclastos reduciendo el MDO, mientras el acetaminofén no afectó el movimiento. ${ }^{6}$

Los objetivos del presente estudio fueron: 1) Determinar los efectos de diclofenaco, etoricoxib, ibuprofeno $y$ paracetamol sobre la magnitud del movimiento dentario ortodóntico en ratas; 2) Evaluar histológicamente las diferencias en la reabsorción ósea en el área de presión producida por el movimiento dentario ortodóntico, en ratas tratadas con diclofenaco, etoricoxib, ibuprofeno y paracetamol.

\section{Materiales y Método}

Participaron 50 ratas machos raza Holtzman de $250 \pm 20$ g, adquiridas en el Bioterio del Instituto Nacional de Salud, que se ambientaron por 7 días en el Bioterio de la Facultad de Medicina de la UNMSM, a una temperatura ambiente entre $19^{\circ}$ a $22^{\circ} \mathrm{C}$, en períodos de luz/oscuridad de 12 horas suministrándoles agua y alimentación balanceada ad libitum.

Se asignaron las ratas en 05 grupos de 10 integrantes cada uno: 01 grupo control (grupo A) y 04 grupos experimentales (grupos B, C, D, E). Todas las ratas fueron anestesiadas con pentobarbital (HALATAL) a dosis de $0.1 \mathrm{~mL} / 250 \mathrm{~g}$ de peso. Los incisivos maxilares de todas las ratas fueron desgastados en su superficie mesial, de vestibular hacia palatino, con una fresa diamantada de fisura de $0.5 \mathrm{~mm}$ de diámetro Fig 1 .

El dispositivo ortodóntico utilizado fue un doblez de 3 vueltas, de $2 \mathrm{~mm}$ de diámetro, con brazos de $9 \mathrm{~mm}$ de longitud, de alambre 0.016 de aleación beta-titanio, con una fuerza recíproca de $35 \mathrm{~g}$. Los brazos del dispositivo pasaron por el tercio cervical, de palatino hacia vestibular, sobre los incisivos maxilares y se adhirieron con una capa de resina (RESILIENCE ORTHOTECNOLOGY). El dispositivo se mantuvo en esa posición por 7 días. Antes de colocar los dispositivos se registró la distancia entre los ángulos incisodistales con un calibrador de precisión de $0.01 \mathrm{~mm}$ (MITUTOYO); luego se midió al $1^{\text {er }}, 3^{\text {er }}, 5^{\text {to }}$ y $7^{\mathrm{mo}}$ día posteriores a la colocación del dispositivo. Las medidas las realizaron 2 observadores, quienes registraron el promedio de 3 mediciones.

Se administraron los fármacos vía intraperitoneal durante 5 días, siendo la primera aplicación inmediatamente después de colocar los dispositivos ortodónticos. El esquema de administración fue el siguiente:

Grupo A: $9 \mathrm{mg} / \mathrm{kg}$ de $\mathrm{NaCl} \mathrm{c/12}$ horas. Grupo B: 10 mg/kg de diclofenaco c/12 horas.

Grupo C: $50 \mathrm{mg} / \mathrm{kg}$ de etoricoxib c/24 horas.

Grupo D: $30 \mathrm{mg} / \mathrm{kg}$ de ibuprofeno c/12 horas.

Grupo E: $200 \mathrm{mg} / \mathrm{kg}$ de paracetamol c/12 horas.

El $1^{\text {er }}, 3^{\text {er }}, 5^{\text {to }}$ y $7^{\text {mo }}$ día luego de la colocación del dispositivo se sacrificaron 2 ratas por cada grupo para realizar los análisis histológicos. Los análisis histológicos estuvieron focalizados en el hueso alveolar interincisivo desde la cresta alveolar hasta los ápices dentarios. El criterio histológico para identificar los osteoclastos fue la presencia de células multinucleadas en la superficie del hueso.

Los análisis estadísticos se realizaron con el SPSS versión 19.0. Se realizó la prueba no paramétrica de Kruskall-Wallis para determinar si se observaron diferencias estadísticamente significativas entre los grupos experimentales $(\mathrm{p}<0.05)$.

Fig. 1 Proceso de Colocación del Dispositivo Ortodóntico
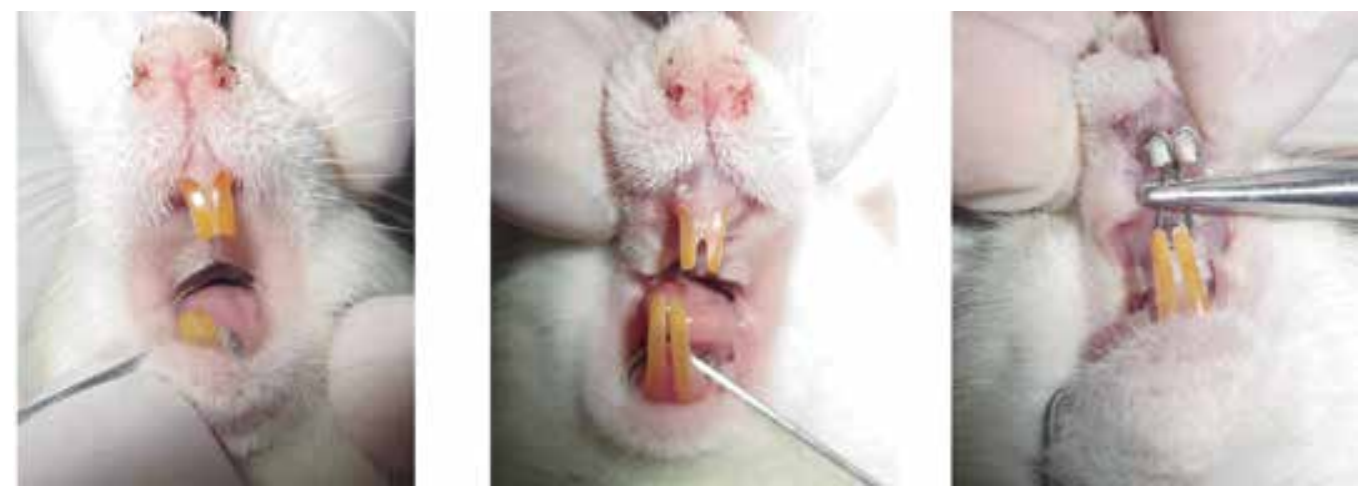


\section{Resultados}

Tabla 1. Distancia interincisiva según tratamiento por días de control

\begin{tabular}{cccccccc}
\hline & $\mathbf{N}$ & Control & Diclofenaco & Etoricoxib & Ibuprofeno & Paracetamol & $\mathbf{P}$ \\
\hline Día 0 & 50 & $2,51 \pm 2,83$ & $2,52 \pm 0,18$ & $2,50 \pm 0,11$ & $2,51 \pm 0,14$ & $2,50 \pm 0,13$ & 0,995 \\
Día 1 & 50 & $4,07 \pm 0,14$ & $3,68 \pm 0,12$ & $3,89 \pm 0,2$ & $3,38 \pm 0,11$ & $4,07 \pm 0,17$ & 0,00 \\
Día 3 & 40 & $4,65 \pm 0,22$ & $4,25 \pm 0,93$ & $4,48 \pm 0,26$ & $3,94 \pm 0,13$ & $4,63 \pm 0,12$ & 0,00 \\
Día 5 & 30 & $5,37 \pm 0,32$ & $4,91 \pm 0,17$ & $5,05 \pm 0,19$ & $4,32 \pm 0,18$ & $5,35 \pm 0,89$ & 0,00 \\
Día 7 & 20 & $5,75 \pm 0,14$ & $5,34 \pm 0,52$ & $5,52 \pm 0,11$ & $4,61 \pm 0,85$ & $5,75 \pm 0,91$ & 0,02 \\
\hline (Kruskall-Wallis, $p<0,05)$ & & & & &
\end{tabular}

En todas las evaluaciones realizadas luego de la colocación del dispositivo ortodóntico (día 1, día 3, día 5, día 7), se evidenciaron diferencias significativas en las distancias interincisivas de las ratas entre los 5 grupos investigados.

Tabla 2. Número de osteoclastos por campo según grupo de tratamiento

\begin{tabular}{cccccccc}
\hline & $\mathbf{N}$ & Control & Diclofenaco & Etoricoxib & Ibuprofeno & Paracetamol & $\mathbf{P}$ \\
\hline Día 1 & 10 & 5 & 4 & 5 & 4 & 5 & 0,00 \\
Día 3 & 10 & 9 & 5 & 6 & 4 & 8 & 0,00 \\
Día 5 & 10 & 12 & 5 & 8 & 5 & 10 & 0,00 \\
Día 7 & 10 & 10 & 6 & 9 & 5 & 12 & 0,02 \\
\hline (Kruskall-Wallis, $p<0,05)$ & & & & & &
\end{tabular}

$\mathrm{Al} 3^{\mathrm{er}}, 5^{\text {to }}$ y $7^{\mathrm{mo}}$ días posteriores a la colocación del dispositivo ortodóntico, se observó una significativa menor cantidad de osteoclastos en el área de presión en los grupos ibuprofeno y diclofenaco en comparación con los grupos etoricoxib, paracetamol y control.

\section{Discusión}

Se comprobó que antes de iniciar el tratamiento (Día 0) no existían diferencias significativas entre las distancias interincisivas de las ratas de los 5 grupos (Tabla 1). Por lo tanto, las diferencias encontradas en los siguientes días de evaluación podrían considerarse relacionadas a efectos producidos por los fármacos administrados.

La disminución en el número de osteoclastos y en el movimiento ortodóntico en los incisivos de las ratas de los grupos ibuprofeno y diclofenaco en comparación al grupo control, observadas en la presente investigación, son similares a lo reportado por Arias y Marquez-Orozco $^{6}$, Bartzela et $\mathrm{al}^{8}$ y De Carlos et $\mathrm{al}^{13}$.

Estudios realizados por Knop et al. ${ }^{14}$ sugieren que la inhibición de las prostaglandinas por los AINEs llevan a la reducción en el número de osteoclastos, lagunas de Howship y vasos sanguíneos en todos los períodos de tratamiento. Retamoso et al. ${ }^{15}$ demostraron una menor tasa de maduración del colágeno en el ligamento periodontal de animales tratados con AINEs y esteroides, con efectos sobre las células relacionadas a la remodelación del hueso y el ligamento periodontal. ${ }^{16,17,18}$
Por otra parte, no se evidenció diferencia significativa en el número de osteoclastos, (Tabla 2) ni en la magnitud del movimiento ortodóntico en el grupo etoricoxib en comparación al grupo control. Resultados similares encontraron De Carlos et al. ${ }^{19}$ y Stabile et al. ${ }^{4}$, quienes utilizando celecoxib demostraron que no se modificó significativamente el movimiento dentario en comparación al grupo control. Resultados distintos a lo encontrado por De Carlos et al. ${ }^{13}$ (utilizando rofecoxib) y Gameiro et al. ${ }^{20}$ (utilizando celecoxib), quienes si encontraron disminución significativa del movimiento ortodóntico en un modelo de mesialización de primeras molares en ratas, en comparación al grupo de ratas sin medicación.

En la presente investigación se observó que no hubo diferencia significativa en el número de osteoclastos, ni en la magnitud del movimiento ortodóntico en los incisivos de las ratas del grupo paracetamol en comparación al grupo control, coincidiendo con Arias y MarquezOrozco $^{6}$, Zambrano ${ }^{21}$, Stabile et al. ${ }^{4}$ y Bartzela et al. ${ }^{8}$. Esto puede ser explicado debido a que el paracetamol actúa sobre el sistema nervioso central sin inhibir la producción periférica de prostaglandinas. Existen diferencias entre el meca- nismo de acción de los AINEs y el paracetamol. Mientras los AINEs bloquean COX-1 y COX-2, el paracetamol bloquea una tercera isoforma, la COX-3, la cual se expresa solamente en cerebro y médula espinal. Como consecuencia el paracetamol tiene efectos mínimos sobre la síntesis periférica de prostaglandinas. ${ }^{8}$ Esto explica que el número de osteoclastos en el área de presión de los incisivos movidos ortodónticamente en el grupo paracetamol, sea muy similar a los observados en el grupo control.

Muchas investigaciones reportadas han concentrado su atención en la inhibición de las prostaglandinas, sin considerar que existen otros mediadores que participan en el movimiento ortodóntico como los leucotrienos, interleuquinas, adenosina monofosfato cíclico (AMPc), guanosina monofosfato cíclico (GMPc) y calcio. Las prostaglandinas tienen un rol importante en el proceso de mecanotransducción, inflamación y dolor, pero su participación es de corta duración, y se produce en presencia de otros mediadores. Con estos conceptos, los investigadores han propuesto la administración a corto plazo de paracetamol, para que actúe como modulador de la producción de prosta- 
glandinas a nivel del Sistema Nervioso Central, ayudando a controlar el dolor antes que inhibir la síntesis periféricas de prostaglandinas, mientras otros mediadores de la reabsorción ósea continúan operando normalmente en los tejidos periodontales. ${ }^{16}$

\section{Conclusiones}

Se demostró que la administración de ibuprofeno y diclofenaco disminuyen significativamente la magnitud del movimiento dentario ortodóntico en ratas.

Se demostró que la administración de etoricoxib y paracetamol no disminuyen significativamente la magnitud del movimiento dentario ortodóntico en ratas.

Fuente de Financiamiento: Vicerrectorado de Investigación de la UNMSM

\section{Referencias Bibliográficas}

1. Krishnan V, Davidovitch Z. Cellular, molecular, and tissue-level reactions to orthodontic force. Am J Orthod Dentofacial Orthop 2006;129(4):469.e1-32.

2. Vargas del Valle P, Piñeiro MS, Palomino H, Torres MA. Factores modificantes del movimiento dentario ortodóntico. Av. Odontoestomatol 2010;26(1):45-53.

3. Campanatti P, Starobinas N, Pallos D. Juveniles versus adults: differences in PGE2 levels in the gingival crevicular fluid during orthodontic tooth movement. Braz Oral Res 2010;24(1):108-13.

4. Stabile AC, Stuani MBS, LeitePanissi CRA, Rocha MJA. Effects of short-term acetaminophen and celecoxib treatment on orthodontic tooth movement and neuronal activation in rat. Brain Research Bulletin 2009;79:396-401.

5. Viecilli RF, Katona TR, Chen J, Hartsfield JK, Roberts WE. Three-dimensional mechanical environment of orthodontic tooth movement and root resorption. Am J Orthod Dentofacial Orthop 2008;133(6):791.e11-26.

6. Arias OR, Marquez-Orosco MC. Aspirin, acetaminophen, and ibuprofen: Their effects on orthodontic tooth movement. Am J Orthod Dentofacial Orthop. 2006;130:364-370.

7. Patel S, Mc Gorray SP, Yezierzki R, Fillingim R, Logan $\mathrm{H}$, Wheeler TT. Effects of analgesics on orthodontic pain. Am J Orthod Dentofacial Orthop 2011;139(1):e53-8.

8. Bartzela T, Turp JC, Motschall SC, Maltha JC. Medication effects on the rate of orthodontic tooth movement: A systematic literature review. Am J Orthod Dentofacial Orthop. 2009;135:16-26.

9. De Carlos F, Cobo J, Diaz-Esnal B, Arguelles J, Vijande M. Orthodontic tooth movement after inhibition of cyclooxygenase- 2 . Am J Orthod Dentofacial Orthop. 2006;129:402-6.

10. De Carlos F, Cobo J, Perillan C, García MA, Arguelles J, Vijande $\mathrm{M}$, et al. Orthodontic tooth movement after different coxib therapies. European Journal of Orthodontics 2007;29:596-9.

11. Gameiro GH, Nouer DF, PereiraNEto JS, Siqueira VC, Andrade ED, Duarte-Novaes P, et al. Effects of short and long-term celecoxib on orthodontic tooth movement. Angle Orthodontist 2008;78(5):860-5.

12. Consolaro A. Analgésicos e antiinflamatórios na movimentação dentária induzida: metodologia e interpretaçáo. Você sabe o que é extrapolação alométrica?. R Dental Press Ortodon Ortop Facial. 2007;12(3):19-23.

13. De Carlos F, Cobo J, Diaz-Esnal B, Arguelles J, Vijande M, Costales
M. Orthodontic tooth movemente after inhibition of cyclooxygenase-2. Am J Orthod Dentofacial Orthop. 2006;129:402-6.

14. Knop LA, Shintcovsk RL, Retamoso LB, et al.Nonsteroidal and steroidal anti-inflamatory use in the context of orthodontic movement. Eur J Orthod. 2011;34:531-5.

15. Retamoso L, Knop L, Shintcovsk $\mathrm{R}$, et al: Influence of anti-inflammatory administration in collagen maturation process during orthodontic tooth movement. Microsc Res Tech. 2011;74:709-13.

16. Krishnan V, Vijayaraghavan N, Manoharan M, Raj J, Davidovitch Z. The Effects of Drug Intake by Patients on Orthodontic Tooth Movement. Semin Orthod. 2012;18:278-85.

17. Karras JC, Miller JR, Hodges JS, Beyer JP, Larson BE. Effect of alendronate on orthodontic tooth movement in rats. Am J Orthod Dentofacial Orthop. 2009;136:843-7.

18. Matsumoto T, Limura T, Ogura K, Moriyama K, Yamaguchi A. The role of osteocytes in bone resorption during orthodontic tooth movement. J Dent Res. 2013;94:340-5.

19. De Carlos F, Cobo J, Perillan C, García MA, Arguelles J, Vijande $\mathrm{M}$, et al. Orthodontic tooth movement after diferent coxib therapies. Eur J Orthod. 2007 Dec;29(6):596-9.

20. Gameiro GH, Nouer DF, Pereira Neto JS, Siqueira VC, Andrade ED, Duarte Novaes P, et al. Effects of short- and long-term celecoxib on orthodontic tooth movement. Angle Orthod. 2008;78:860-5.

21. Zambrano DC. Acetaminofén con codeína eficiente contra el dolor dentario. Ciencia UAT. 2008 Dic;48-51. 